\title{
Heart rate, skin potential response, and latency of overt response, as indicators of problem recognition and solution
}

\author{
MARJORIE F. ELIAS, Larsen Hall, \\ Harvard University, Cambridge, Mass. \\ 02138
}

Heart rate, skin potential, and response latency were studied in relation to the recognition and solution of problems by 18 high-school students. $A$ continuous series of rule-induction problems was presented without any pause between problems. The problems were sequentially related in that each new problem demanded a refinement of the rule just induced for the preceding problem. Increases in skin potential and response latency occurred when new problems were introduced, and decreases in the same measures were found when they were solved. Heart rate was not indicative of recognition or solution of problems. When events during trials-stimulus, response, feedback-were looked at separately, the events of stimulus onset and response were associated with significant increases at recognition. Only the feedback event was associated with a significant decrease at solution. It was suggested that these changes indicate a labile state during which problem solving occurs.

Decreases in skin-potential response and latency of overt response occurred at solution of problems in a previous study (Elias \& White, 1969). This result led to the speculation that these responses might subsequently increase when a new problem was recognized and diminish when it, in turn, was solved. The present study was designed to test response to recognition as well as solution of a problem. Changes in response in detail were also studied by separating events during trials and analyzing trial-by-trial changes.

Problem recognition, as well as solution, was introduced by designing tasks that presented a series of problems requiring increasing refinement of a rule for solution in an uninterrupted sequence of trials. Slides that required a choice among four items were presented. In order to choose correctly, the $\mathbf{S}$ had to induce a rule, such as "the even number." When a rule had been successfully learned, an anomalous slide was presented with two items correct by the rule. A refinement of the rule was now required in order to distinguish which of the two items was correct. The term "anomaly" is defined as a phenomenon that cannot be accounted for by the rules currently operating. In the continuing stream of experience, recognition of anomaly would seem to be a necessary condition for the solution of a problem. One must recognize that one has a problem in order to solve it.

In order to determine more precisely what accounted for the changes at recognition and solution of problems, the study separated response to events in each trial: slide presentation, button-press response with which the $S$ indicated his choice, and onset of a light telling him which choice was, in fact, correct. This procedure was suggested by Germana (1968) in a recent review of studies on physiological correlates of learning. In the same article, Germana postulated an activational peak at the time of acquisition of a conditioned response. A trial-by-trial analysis was made in the present study in order to test Germana's hypothesis on a more complex type of learning task.

The response systems being studied were heart rate, skin potential, and response latency. Skin-potential response and latency of overt response were used because they had been found to show reliable changes in the earlier study cited above. Heart rate was included because of indications in the literature that suggested that it might vary with learning. Deceleration in anticipation of a signal has been demonstrated by Lacey (1965). Since such decelerations might vary in amplitude with recognition and solution of problems, it was decided to measure decelerations in anticipation of the S's button press and the feedback light. Blatt (1961) reported accelerations at key points during the process of problem solving. Accordingly, we measured accelerations as responses after each event in a trial.

It was expected that some of the response systems would show increased reactivity at recognition and decreases at solution of a problem, and that some events during trials might contribute more than others to such changes.

\section{METHOD}

The Ss were 20 9th- and 10th-grade students from the college preparatory track of a large city high school. Two records were not scorable for technical reasons, so results are based on 18 records from 10 girls and 8 boys.

The task format was unique in that the tasks were designed in stages but presented in an unbroken series of trials. The $S$ could perceive the shifts from stage to stage only if he solved each problem and recognized the anomalous character of a slide at the beginning of a new problem. The rules were as follows. Number task: (1) even number, (2) two-digit even number, (3) two-digit even number of 50 or higher, (4) two-digit even number of 50 or higher that was a multiple of 10 . Word task: (1) verb, (2) past-tense verb, (3) past-tense verb ending in $d$, (4) five-letter past-tense verb ending in $d$. The rules became more complex in statement at each successive stage, but subjectively Ss probably experienced each new rule refinement as an inductive problem of about the same difficulty as the problem previously solved.

Trials were given according to the first rule until the $S$ reached a criterion of six consecutive correct responses. The next slide presented was anomalous by the first rule in that two items were correct. To choose between those two items, a refinement of Rule 1, namely Rule 2, was needed. Trials governed by the second rule were presented until that was solved by the criterion of six consecutive correct choices. In the same way, Rule 3 and then Rule 4 were presented and solved. If the $S$ failed at any stage to reach criterion in 18 trials, he was told the rule for that stage. He was then given six more trials at the same stage to which he could respond correctly before going on to the next stage. Each trial started with presentation of a slide showing four items. The $S$ indicated his choice by pressing one of four buttons at his own pace. A feedback light came on $7.5 \mathrm{sec}$ after the button press to show which choice was, in fact, correct, regardless of which choice had been made. The next trial was initiated by the $E$ after an interval of at least $8 \mathrm{sec}$. The intervals were planned to allow time for physiological response to each event.

Measures were recorded on paper output of a Grass Model 7 polygraph. Heart rate was recorded through a cardiac tachometer taken from left-arm/right-arm or left-arm/right-leg electrode placement. Skin potential was recorded as a dc measure from silver-silver chloride electrodes placed on the palm of the nondominant hand with reference to the back of the wrist. Events of slide onset, button press, and light onset were marked on the record. Response latency was measured as millimeters from slide onset to button press on paper moving at $3 \mathrm{~mm} / \mathrm{sec}$. The procedure was programmed on Grason-Stadler Series 1200 modules.

After electrodes were applied, the S was seated at a table facing the screen and the panel of response buttons and feedback lights. His back was turned to $\mathrm{E}$ and the 
polygraph. He was instructed that he was to find a rule and then refine it in stages. A preliminary practice task was given. Its rules were then explained to him and the increasing refinement required was pointed out. He was then given one task, a rest period of about $2 \mathrm{~min}$, and then the other task. The order of the tasks was alternated among Ss. The session lasted $1 \mathrm{~h}$.

Autonomic records were scored as follows. Heart-rate responses to events were measured by subtracting the previous level (mean of the three beats before slide onset) from the maximum beat in the $4 \mathrm{sec}$ following each event. Anticipatory responses were measured by subtracting the minimum beat in the 4-sec period before an event from the same previous level. The total heart-rate response to a trial was the range between the highest maximum and the lowest minimum beat on that trial.

Skin-potential responses were scored as millimeters of pen deflection occurring in the 4-sec period after each event in a trial. The total skin-potential response to a trial was the sum of the three responses to parts of the trial.

Heart-rate and skin-potential scores were transformed by dividing each score by S's standard deviation of scores in order to make scores of different Ss comparable in variability. Response latency was transformed by a log transformation to normalize the distribution.

Three statistical analyses were made of the scores to test for increases at recognition, decreases at solution, and trial-by-trial trends.

The prediction of increase at recognition of anomaly was tested by finding the difference between each response on an anomalous trial and response to the same event on the preceding trial. Differences would average out to approximately zero unless there were significantly more response to anomaly; so matched $t$ tests were performed to test the significance of the mean difference with reference to zero.

To test the prediction of decrease at solution, three 10-trial sequences were isolated from the unbroken series of trials for each $S$ in the following way. Two of the sequences, called solved sequences, were 10 trials of which the last 6 were all correct. In other words, solution could be said to have occurred on the fifth trial. These could be divided into 5 trials before and including solution and 5 trials after solution, so mean response amplitudes before and after solution could be compared. Sequences that were solved spontaneously were used for analysis, but it should be mentioned that when the $S$ was told the rule, his change in response pattern was not noticeably different. The third 10-trial sequence, the control sequence, was chosen from a part of the record in which 10 consecutive trials occurred without solution taking place. These 10 trials could also be divided into 5 and 5. This controlled for the effects of changes over time independent of solving. Change scores were calculated by subtracting the mean of the 5 responses to an event after solving (or late in the control sequence) from the mean before solving (or early in the control sequence). A mean change-solved score for each $S$ was found by averaging the two scores from his solved sequences. Changes during solved and during control sequences were each tested by matched $t$ test. The difference between each S's solved and control sequence changes was also tested.

Comparisons were made separately for skin potential and heart rate for each event during a trial: response to slide onset, anticipation of button press (heart rate only), response to button press, anticipation of feedback light (heart rate only), response to feedback light; and also for overall response to the trial: heart-rate range, skin-potential sum of responses to the events during the trial, and response latency. Forty comparisons were made in all.

A trial-by-trial analysis was made of the 10 trials in solved and control sequences for skin-potential and latency measures. A mean transformed score for all 18 Ss for each event in each trial in the sequence was found.

\section{RESULTS AND DISCUSSION}

Increases at recognition and decreases at solution were found in the measures of skin potential and latency of overt response. Heart rate showed no changes related to problem recognition or solution. Observed changes and $t$ tests of significance are given in Table 1. Total skin-potential response and latency of overt response were found to increase strongly at recognition of a problem. They also decreased at its solution, confirming our findings noted earlier, although the difference between decreases in control and solved sequences did not reach significance for the total skin potential measure in the present study. These results suggest that skin-potential response and latency of overt response might be useful as indicators of recognition and solution of problems.

Looking at skin-potential response to events during trials, a contrast can be seen between problem recognition and solution. At recognition of a problem, increases in response occur to slide presentation and to pressing the button, whereas, at solution, the significant decrease is to the feedback light. Both findings can be interpreted in

Table 1

Significance of Change in Response Amplitude at Recognition and Solution of Problems

\begin{tabular}{|c|c|c|c|c|c|c|c|c|}
\hline \multirow[b]{3}{*}{ Response } & \multirow{2}{*}{\multicolumn{2}{|c|}{$\begin{array}{c}\text { Increase at } \\
\text { Recognition } \\
\text { of a Problem } \\
\end{array}$}} & \multicolumn{6}{|c|}{$\begin{array}{c}\text { Decrease at } \\
\text { Solution of a Problem }\end{array}$} \\
\hline & & & \multirow{2}{*}{\multicolumn{2}{|c|}{$\frac{\text { Mean Solved Sequence }}{\text { Mean }}$}} & \multicolumn{2}{|c|}{ Control Sequence } & \multicolumn{2}{|c|}{ Solved-Control } \\
\hline & $\begin{array}{c}\text { Mean } \\
\text { Increase }\end{array}$ & $\mathbf{t}$ & & & $\begin{array}{c}\text { Mean } \\
\text { Decrease }\end{array}$ & $\mathrm{t}$ & $\begin{array}{c}\text { Mean } \\
\text { Difference }\end{array}$ & $\mathbf{t}$ \\
\hline \multicolumn{9}{|l|}{ Heart Rate (in Transformed Scores) } \\
\hline Response to Slide & 0.04 & 0.40 & 0.03 & 0.32 & -0.02 & -0.13 & 0.05 & 0.22 \\
\hline Anticipatory Response to Button & see note & & -0.11 & -0.93 & -0.10 & -0.56 & -0.01 & -0.03 \\
\hline Response to Button & & & 0.02 & 0.12 & 0.19 & 1.06 & -0.17 & -0.57 \\
\hline Anticipatory Response to Light & & & 0.04 & 0.39 & -0.03 & -0.17 & 0.07 & 0.29 \\
\hline Range & 0.11 & 0.82 & -0.01 & -0.16 & 0.11 & 0.79 & -0.12 & -0.63 \\
\hline & & & 0.02 & 0.13 & 0.07 & 0.48 & -0.06 & -0.27 \\
\hline \multicolumn{9}{|l|}{ Skin Potential (in Transformed Scores) } \\
\hline Response to Slide & 0.29 & $2.55^{*}$ & 0.22 & 1.56 & -0.04 & -0.24 & 0.26 & 1.12 \\
\hline Response to Button & 0.37 & $2.56 *$ & 0.22 & 1.33 & 0.33 & 1.53 & -0.11 & -0.38 \\
\hline Response to Light & 0.04 & 0.20 & 0.34 & $2.87^{*}$ & -0.14 & -0.94 & 0.48 & $2.89 *$ \\
\hline Total Response & 0.80 & $4.12 * * *$ & 0.77 & $2.44^{*}$ & 0.15 & 0.44 & 0.61 & 1.43 \\
\hline Response Latency (in Transformed Scores) & 0.16 & $6.41 * * *$ & 0.13 & $7.61 * * *$ & 0.03 & 1.14 & 0.10 & $3.13^{* *}$ \\
\hline
\end{tabular}

Note: Analyses were not made for some heart rate increases because all previous heart rate analyses had been negative.

$* p<.05 \quad p<.01 \quad p<.001$ 

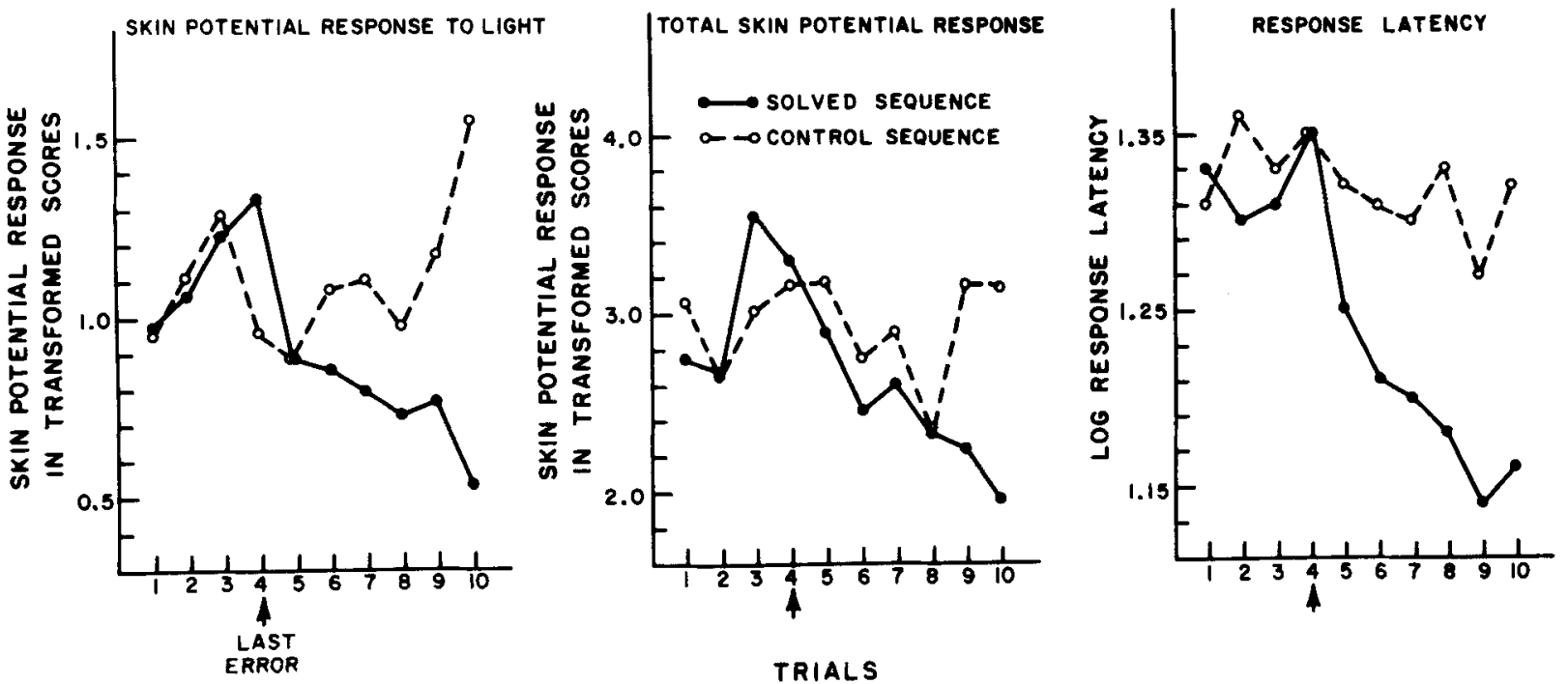

Fig. 1. Trial-by-trial comparison of control and solved sequences in which significant decreases in response occurred at solution.

terms of the S's estimate of the probability of an outcome. Larger response occurs to an outcome that is judged to be less probable. In recognition of a problem, presentation of an anomalous slide was a less probable event than presentation of a slide like those preceding. The event of slide presentation evoked increased response. The change in reaction to button press can be interpreted as being due to continued response to recognition of the unexpected slide as the $\mathrm{S}$ attempts to choose between items not differentiated by his rule. At solution, however, the change in probability was related to the event of feedback, a change from not being sure what its outcome would be to being sure. As the S's estimate of the probability of an outcome increased, the size of his response to the outcome diminished. It is interesting to note that Walter (1966) called his contingent negative variation of frontal EEG (CNV) an "expectancy wave" because he interpreted it in terms of the S's assessment of the probability of an outcome. It varies directly rather than inversely with expectancy, however, and occurs before the outcome.

An alternative explanation of the decrease in responsivity to the feedback light at solution might be that there were larger responses to being proven wrong than to being right. This would result in a decrease at solution since there are no errors after solution. But this explanation is not consistent with the finding of no difference $(t=-0.02, p=1.0)$ between response to the feedback light in incorrect and correct trials before solution. This result strengthens the inference that it is an outcome that has been subjectively judged to be improbable rather than one that is objectively proven to be wrong that contributes to the skin-potential response.

Decreases are evident in solved sequences after solution in trial-by-trial graphs of sequences of the response systems that showed significant changes at solution. Germana's (1968) activational peak is evident in each graph, but the rise before solution is less strong than the decline afterwards. Only in the postsolution decrease does the solved sequence separate clearly from the control sequence. The early rise may be attributable to variability in the presolution phase rather than to a significant upward trend.

The findings reported here show changes in skin potential and response latency at recognition and solution of problems, but not in heart rate. The results are consistent with our earlier findings, which showed equivalent changes in skin potential but were negative for vasoconstriction of the finger. No explanation is offered for the lack of reliable changes in the cardiovascular system. Studies of other autonomic responses during problem solving do show comparable changes, however. Malmo (1965) has found increased muscle tension. Kahneman (1967) reports dilation of the pupil of the eye. It seems that, when a situation arises for which one does not have a confident prediction of outcome or a ready plan for response, one enters a state that is characterized by increased autonomic reactivity, longer response time, and a subjective sense of uncertainty. It is suggested that in this open labile state, learning can take place.

\section{REFERENCES}

BLATT, S. J. Patterns of cardiac arousal during complex mental activity. Journal of Abnormal \& Social Psychology, 1961, 63, 272-282.

ELIAS, M. F., \& WHITE, S. H. Some psychophysiological and latency correlates of rule induction. Psychonomic Science, 1969 , $16,84-85$

GERMANA, J. Psychophysiological correlates of conditioned response formation. Psychological Bulletin, 1968, 70, 105-114.

KAHNEMAN, D., \& BEATTY, J. Pupillary responses in a pitch-discrimination task. Perception \& Psychophysics, 1967, 2, 101-105.

LACEY, B. C., \& LACEY, J. I. Cardiovascular and respiratory correlates of reaction time. Progress Report MH00623, Fels Research Institute, Yellow Springs, Ohio, June 1965.

MALMO, R. B. Physiological gradients and behavior. Psychological Bulletin, 1965, 64, 225-234.

WALTER, W. G. The relations between electrical signs of expectancy in the human brain and autonomic function during operant and classical conditioning. Abhandlungen der deutschen Akademie der Wissenschaften zu Berlin, 1966, 2, 47-55.

NOTE

1. This research was supported by Grant OE 5-10-239 from the Office of Education. Thanks are due to Sheidon $H$. White and Bernard Tursky for their help with the experimental design and instrumentation of this study. 